\title{
A feedback fluid queue with two congestion control thresholds
}

\author{
R. Malhotra - M. R. H. Mandjes • \\ W. R. W. Scheinhardt • J. L. van den Berg
}

Received: 31 October 2007 / Accepted: 22 May 2008 / Published online: 2 September 2008

(C) The Author(s) 2008. This article is published with open access at Springerlink.com

\begin{abstract}
Feedback fluid queues play an important role in modeling congestion control mechanisms for packet networks. In this paper we present and analyze a fluid queue with a feedback-based traffic rate adaptation scheme which uses two thresholds. The higher threshold $B_{1}$ is used to signal the beginning of congestion while the lower threshold $B_{2}$ signals the end of congestion. These two parameters together allow to make the trade-off between maximizing throughput performance
\end{abstract}

R. Malhotra

Alcatel-Lucent, Capitool 5, 7521 PL Enschede, The Netherlands

e-mail: rimalhotra@alcatel-lucent.com

R. Malhotra · J. L. van den Berg

University of Twente, 7500 AE Enschede, The Netherlands

M. R. H. Mandjes

Korteweg-de Vries Institute for Mathematics, Plantage Muidergracht 24, 1018 TV Amsterdam, The Netherlands

e-mail: mmandjes@science.uva.nl

M. R. H. Mandjes · W. R. W. Scheinhardt

CWI, Amsterdam, The Netherlands

M. R. H. Mandjes

EURANDOM, Eindhoven, The Netherlands

W. R. W. Scheinhardt

Faculty of Electrical Engineering, Mathematics, and Computer Science,

University of Twente, P.O. Box 217, 7500 AE Enschede, The Netherlands

e-mail: w.r.w.scheinhardt@utwente.nl

J. L. van den Berg $(\bowtie)$

TNO ICT, P.O. Box 5050, 2600 GB Delft, The Netherlands

e-mail: j.1.vandenberg@tno.nl 
and minimizing delay. The difference between the two thresholds helps to control the amount of feedback signals sent to the traffic source. In our model the input source can behave like either of two Markov fluid processes. The first applies as long as the upper threshold $B_{1}$ has not been hit from below. As soon as that happens, the traffic source adapts and switches to the second process, until $B_{2}$ (smaller than $B_{1}$ ) is hit from above. We analyze the model by setting up the Kolmogorov forward equations, then solving the corresponding balance equations using a spectral expansion, and finally identifying sufficient constraints to solve for the unknowns in the solution. In particular, our analysis yields expressions for the stationary distribution of the buffer occupancy, the buffer delay distribution, and the throughput.

Keywords Fluid queues · Feedback regulation - Congestion control · Spectral expansion

\section{Introduction}

In performance analysis of communication systems, fluid queues are a frequently used modeling framework. These models approximate packet streams by flows of fluid. Feedback fluid queues are of special significance when modeling congestion control mechanisms, in particular closed-loop controls in which the input process is affected by the current value of the buffer content. Practical examples are TCP congestion control, random early detection (Floyd and Jacobson 1993), explicit congestion notification (Floyd 1994) and Ethernet congestion control mechanisms (Malhotra et al. 2005).

Previous work on feedback fluid queues (Mandjes et al. 2003) predominantly focused on queues in which a single buffer threshold signals both the onset and end of congestion. In these models, depending on whether the buffer occupancy is below or above the threshold, the traffic source is allowed to transmit at a high peak rate or slowed down to a (lower) guaranteed traffic rate, respectively. The special case at which the threshold lies at 0 was also addressed in, e.g., (da Silva Soares and Latouche 2005; Ramanan and Weiss 1997).

These 'single-threshold systems' have serious drawbacks. In the first place, in case the threshold is crossed often, many feedback signals have to be sent to the traffic source, and these may consume a significant part of the available bandwidth. In the second place, it can be argued that it is not optimal that a single threshold performs the function of signaling the beginning and end of congestion: to maximize throughput a full buffer is preferred (thus delaying the signal for the onset of congestion as much as possible), whereas to minimize the buffer delay requires minimizing the buffer occupancy (thus delaying the signal for the end of the congestion phase as much as possible). Therefore, to find a good trade-off between throughput and buffer delay, there is a need for mechanisms with two separate thresholds: one to signal the beginning of the congestion phase, and an other to signal the end. In this paper we propose and analyze such a mechanism.

The model addressed in this paper belongs to the class of (Markovian) fluid models. The 'classical' fluid model (Anick et al. 1982; Kosten 1984) is characterized by a generator matrix $Q$ governing a Markovian background process and a diagonal matrix 
$R=\operatorname{diag}\left\{r_{1}, \ldots, r_{d}\right\}:$ if the background process is in state $i$, traffic is generated at a rate $r_{i} \geq 0$. It was shown that the steady-state buffer content distribution obeys a system of linear differential equations, and after imposing the proper additional constraints these can be solved. From a methodological standpoint, an important contribution was due to Rogers (1994), who succeeded to express the steady-state buffer content distribution in terms of the fundamental Wiener-Hopf factorization. Another key paper is by Ahn and Ramaswami (2005), who explicitly exploit relations with quasi-birthdeath processes. We also mention that a nice (recent) literature overview on Markov fluid queues is given in, e.g., da Silva Soares and Latouche (2006).

In the second half of the 1990s models emerged in which the source behavior was influenced by the buffer content; see for instance (Adan et al. 1998; da Silva Soares and Latouche 2005; Mandjes et al. 2003; Ramanan and Weiss 1997); in Gribaudo and Telek (2007); Scheinhardt et al. (2005), $Q$ and $R$ depend continuously on the buffer level. Importantly, in all these feedback fluid models the buffer content uniquely defines the probabilistic properties of the source. The model analyzed in the present paper departs from this property. In fact the input process has two 'modes'. The first mode applies as long as the upper threshold $B_{1}$ has not been reached from below. As soon as that happens, we switch to the second mode, until the lower threshold $B_{2}$, smaller than $B_{1}$, is hit from above, i.e., the buffer occupancy falls below $B_{2}$. In this way the threshold $B_{1}$ is used to signal the onset of congestion, and $B_{2}$ to signal the end of congestion. Important novelty of our model as compared to earlier feedback models is that the input process may behave in two different ways between the two thresholds, depending on the past.

In this paper our goal is to find the steady-state buffer content distribution and associated performance measures of the model with two thresholds, as was described above. The methodology used involves the derivation of Kolmogorov equations and balance equations, which result in a solution in terms of a spectral expansion; then additional conditions are identified that solve for the unknowns in the solution. Although this method is in principle similar to the approach in, e.g., Mandjes et al. (2003), our model poses new challenges, due to its specific features (i.e., the two thresholds, the two modes of the input process). In the analysis particularly the behavior at the thresholds should be handled with care. As a result, the derivation of the conditions to solve for the unknowns turns out to be substantially more difficult than in the model of Mandjes et al. (2003).

We mentioned above that the model we investigate can be useful in detecting congestion and can be generically applied to any congestion control mechanism for packet networks such as Floyd and Jacobson (1993), Floyd (1994) and Malhotra et al. (2005). Here we explain how this kind of feedback control has special significance with respect to congestion control in Ethernet metropolitan networks. The back-pressure scheme defined in IEEE $802.3 \times$ (1998), is intended to provide flow control on a hop-by-hop basis by allowing ports to turn off their upstream link neighbors for a period of time. For a full-duplex connection, this mechanism is based on a special frame called pause frame in which the pause period is specified. The end-station (or router) receiving the pause frame looks at the pause period, and does not transmit or attempt transmission for that amount of time. Alternatively, an ON/OFF pause message can be sent signaling the beginning and end of the transmission pause phase. This congestion control 
method is usually implemented by using a high and a low threshold in the (congested) queue. When the queue occupancy exceeds the high threshold the PauseOn message is sent and when the queue occupancy drops below the low threshold the PauseOff message is sent and consequently transmission is resumed. Previous works (Malhotra et al. 2002, 2005; Noureddine and Tobagi 1999) on the Ethernet congestion control have concentrated on the throughput gain which can be achieved by using the scheme. We are not aware of any literature with an analytically tractable model of the mechanism. The model presented in this paper can be used to optimally configure the high and low thresholds and decide on when to initiate the transmission pause phase and when to end it, consequently, addressing an essential design criterion for the Ethernet congestion avoidance scheme.

The rest of the paper is organized as follows. In Sect. 2 we describe the model with two thresholds in detail. In Sect. 3 we analyze the model and derive the equilibrium distribution of the buffer content. This section consists of three parts. Section 3.1 gives the balance equations for the buffer occupancy. Section 3.2 uses the spectral expansion method to provide the solution to the buffer occupancy in a compact form, which involves several unknown coefficients. In Sect. 3.3 we derive as many constraints as there are unknowns, so that these coefficients can be identified. In Sect. 4 we demonstrate our analysis by considering a numerical example and graphically present the buffer content distribution; we remark that a full numerical assessment of the back-pressure mechanism, relying on the methods developed in this paper, is found in Malhotra et al. (2008). We do include here, however, numerical evidence for the claim that the two-threshold mechanism leads to a reduction of the signaling overhead. Finally in Sect. 5 we conclude the paper with a summary of our results, and a discussion on future work.

\section{Model}

In this section we provide the formal definition of the model. We consider a fluid queue with an infinite buffer and constant output rate $c$. Let $W(t)$ be the content of the queue at time $t$, which is a stochastic process due to the probabilistic way in which fluid enters the queue. A popular model for such an input process is a so-called Markov fluid source. This model prescribes that the rate at which fluid enters the queue per unit time depends on the current state of some background irreducible continuoustime Markov chain $X(t)$, defined on a finite state-space $\{1, \ldots, d\}$, for $d \in \mathbb{N}$. At times when $X(t)=i$, the current input rate is $r_{i} \geq 0$. When we let the corresponding generator matrix be $Q \equiv\left(q_{i j}\right)_{i, j=1}^{d}$, with $\sum_{j=1}^{d} q_{i j}=0$ and $q_{i j} \geq 0$ for $i \neq j$, and define the $d$-dimensional traffic rate vector $r \equiv\left(r_{1}, \ldots, r_{d}\right)^{\mathrm{T}}$, we call this input process a Markov-fluid source with parameters $Q$ and $\boldsymbol{r}$.

In our feedback fluid model, the input stream alternates between two modes. In one mode the input process behaves like a Markov fluid source with generator $Q^{+}$ (dimension $d \times d$ ) and traffic rate vector $\boldsymbol{r}^{+}$(dimension $d$ ). Similarly, in the other mode it behaves like a Markov fluid source with generator $Q^{-}($also dimension $d \times d)$ and traffic rate vector $\boldsymbol{r}^{-}$(also dimension $d$ ). 


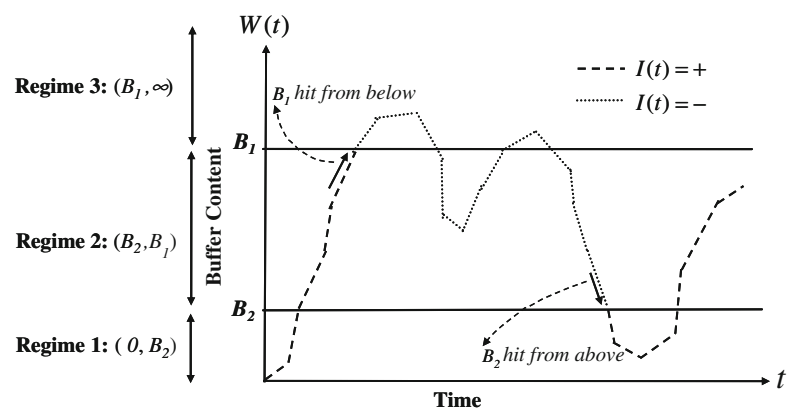

Fig. 1 Different regimes for the buffer content $W(t)$

We introduce the indicator variable process $I(\cdot)$, taking values in $\{$ ' + ', ' - ' $\}$, which gives the current mode of operation of the input source. It is important to note that whenever $I(t)$ switches from one mode to another, the background process $X(t)$ stays in the same state; only its dynamics will from that time onwards behave according to the other generator matrix. However, the rate at which the fluid buffer receives fluid does change instantaneously from $r_{i}^{+}$to $r_{i}^{-}$(or vice versa), when the background process $X(t)$ is in state $i$ at the switching instant. Which of the two modes is currently valid at some time $t$ depends on the behavior of the content process $W(t)$ relative to two thresholds, an upper threshold $B_{1}$ and a lower threshold $B_{2}$. The first mode ('+') applies as long as $W(t)$ has not reached the upper threshold $B_{1}$ from below. As soon as that happens, $I(t)$ switches to the other mode ('-'), until $W(t)$ hits the lower threshold $B_{2}$ from above, etc.

Let us describe this in some more detail, see also Fig. 1 . Suppose $W(0)=0$, i.e., the process starts with an empty buffer, and let the indicator process $I(t)$ start in ' + ', where it will stay as long as the process $W(t)$ has not reached $B_{1}$ from below. During this period the input process behaves as a Markov-fluid source with $d$-dimensional generator $Q^{+}$and traffic rate vector $\boldsymbol{r}^{+}$, and the buffer is drained at a rate $c$. At some point the buffer content $W(t)$ reaches the upper threshold $B_{1}$. Suppose that $X(t)$ is then in state $j$. Then $I(t)$ switches to '-', while the background process $X(t)$ stays in state $j$. From then on the input process behaves as a Markov-fluid source with generator $Q^{-}$and traffic rate vector $\boldsymbol{r}^{-}$, while the buffer is still drained at rate $c$. In particular, the current flow rate will change from $r_{j}^{+}$, which is larger than $c$, to $r_{j}^{-}$, which may or may not be larger than $c$. Further on at some moment the buffer content $W(t)$ drops to the lower threshold $B_{2}$. Suppose that $X(t)$ is in state $k$ at this moment, then $I(t)$ switches to ' + ' while the background process stays in state $k$, and the input rate changes from $r_{k}^{-}<c$ to $r_{k}^{+}$.

Thus, the process continues, and will converge to an equilibrium distribution, assuming the queue is stable. With $\pi^{-}$denoting the equilibrium distribution corresponding to $Q^{-}$, i.e., $\pi^{-} Q^{-}=0$ and $\sum_{i=1}^{d} \pi_{i}^{-}=1$, the equilibrium condition is

$$
\sum_{i=1}^{d} \pi_{i}^{-} r_{i}^{-}<c
$$


throughout this paper we assume that this condition is satisfied. For technical reasons, we will also assume that for all states $i=1, \ldots, d$ we have $r_{i}^{+} \neq c$ and $r_{i}^{-} \neq c$, so that the content of the queue is never constant over time (unless it is zero). Let $W$ be the steady-state buffer content, i.e., a random variable distributed as $\lim _{t \rightarrow \infty} W(t)$ and define $I$ and $X$ similarly. Define also, for $i=1, \ldots, d$ and $x \geq 0$,

$$
F_{i}^{-}(x):=\mathbb{P}(I=-, X=i, W \leq x), \quad F_{i}^{+}(x):=\mathbb{P}(I=+, X=i, W \leq x) .
$$

The goal of our paper is to identify $F_{i}^{-}(x)$ and $F_{i}^{+}(x)$. Having solved for these distribution functions, we can calculate two important performance measures for the system, namely the throughput $\vartheta$ and the distribution of the delay $D$, as follows:

$$
\vartheta=\sum_{i=1}^{d}\left(r_{i}^{+} F_{i}^{+}(\infty)+r_{i}^{-} F_{i}^{-}(\infty)\right)
$$

and for $d \geq 0$,

$\mathbb{P}(D \leq d)=\left(\sum_{i=1}^{d}\left(r_{i}^{+} F_{i}^{+}(d / c)+r_{i}^{-} F_{i}^{-}(d / c)\right)\right) /\left(\sum_{i=1}^{d}\left(r_{i}^{+} F_{i}^{+}(\infty)+r_{i}^{-} F_{i}^{-}(\infty)\right)\right)$.

We define some additional notation which will be helpful in considering the various cases while solving for $F_{i}^{-}(x)$ and $F_{i}^{+}(x)$. We define the sets of 'up-states' and 'downstates' for both modes, and their cardinalities, as follows:

$$
\begin{aligned}
& S_{\mathrm{D}}^{-}:=\left\{i: r_{i}^{-}<c\right\} \text { and } d_{\mathrm{D}}^{-}:=\#\left\{S_{\mathrm{D}}^{-}\right\} ; \\
& S_{\mathrm{U}}^{-}:=\left\{i: r_{i}^{-}>c\right\} \text { and } d_{\mathrm{U}}^{-}:=\#\left\{S_{\mathrm{U}}^{-}\right\} ; \\
& S_{\mathrm{D}}^{+}:=\left\{i: r_{i}^{+}<c\right\} \text { and } d_{\mathrm{D}}^{+}:=\#\left\{S_{\mathrm{D}}^{+}\right\} ; \\
& S_{\mathrm{U}}^{+}:=\left\{i: r_{i}^{+}>c\right\} \text { and } d_{\mathrm{U}}^{+}:=\#\left\{S_{\mathrm{U}}^{+}\right\}
\end{aligned}
$$

The subscript $D$ is a mnemonic for 'Down', referring to the buffer being drained, while $U$ stands for 'Up', referring to the buffer filling up. Evidently, $d_{\mathrm{D}}^{-}+d_{\mathrm{U}}^{-}=d_{\mathrm{D}}^{+}+d_{\mathrm{U}}^{+}=d$.

\section{Analysis}

In this section we analyze the buffer content distribution, by presenting a procedure to compute $F_{i}^{-}(x)$ and $F_{i}^{+}(x)$, for $i=1, \ldots, d$. From the model description in the previous section we know that $F_{i}^{-}(x)$ and $F_{i}^{+}(x)$ have different characteristics in different intervals of the buffer content. Therefore, we define Regimes 1, 2, and 3, as shown in Fig. 1. For each of these regimes we analyze $F_{i}^{-}(x)$ and $F_{i}^{+}(x)$. Two cases are rather straightforward, and therefore we start with these.

$3^{+}: F_{i}^{+}(x)$ in Regime 3 . When the buffer occupancy reaches $B_{1}$ the indicator switches to the '-' state (if it was not in '-' already). The indicator changes to ' + ', only 
after the buffer occupancy would drop below $B_{2}$, where $B_{2}<B_{1}$. Therefore, $F_{i}^{+}(x)$ is constant in the interval $\left[B_{1}, \infty\right)$. For $i=1, \ldots, d$ and $x \geq B_{1}$,

$$
F_{i}^{+}(x)=F_{i}^{+}\left(B_{1}\right)
$$

$1^{-}: F_{i}^{-}(x)$ in Regime 1 . If the buffer occupancy drops below $B_{2}$, then the indicator switches to ' + '. Therefore, the fluid source with the ' - ' indicator can never be active below $B_{2}$ but only in the interval $\left[B_{2}, \infty\right)$. We have, for all $i=1, \ldots, d$ and $x \leq B_{2}$,

$$
F_{i}^{-}(x)=0 \text {. }
$$

Nevertheless, it is important to note that even though $F_{i}^{-}\left(B_{2}\right)=0$ the density at $B_{2}$, i.e.,

$$
f_{i}^{-}\left(B_{2}\right):=\left.\frac{\mathrm{d} F_{i}^{-}(x)}{\mathrm{d} x}\right|_{x \downarrow B_{2}},
$$

might not be equal to zero.

The other cases $1^{+}, 2^{+}, 2^{-}$and $3^{-}$are analyzed in the following subsections. We follow an approach similar to that in Anick et al. (1982) and Mandjes et al. (2003) to find the complete solution to $F_{i}^{-}(x)$ and $F_{i}^{+}(x)$. We derive the balance equations for both $F_{i}^{-}(x)$ and $F_{i}^{+}(x)$ in Sect. 3.1, by first considering the Kolmogorov forward equations. What makes the Kolmogorov equations especially complicated in our case are the transitions around the thresholds $B_{1}$ and $B_{2}$. Assuming that a transition takes place somewhere in a small time interval, the exact time of the transition is unknown, and as a consequence so is the indicator of the generator matrix in that interval. Section 3.1 deals with these issues. In Sect. 3.2 the spectral expansion method is used to find the solution to the differential equations. These can be written down in a rather simple form, but involve an extensive set of unknown coefficients. In Sect. 3.3 we find as many conditions as the number of unknowns, so that the stationary distribution of the buffer content can be determined.

\subsection{Derivation of the balance equations}

We found above simple solutions for $F_{i}^{+}(x)$ in Regime 3 and $F_{i}^{-}(x)$ in Regime 1, given by Eqs. (2) and (3). Our goal in this subsection is to derive the Kolmogorov forward equations for the other cases, from which we then easily obtain the corresponding balance equations. We slightly abuse notation by also using $F_{i}^{-}$and $F_{i}^{+}$for the timedependent distribution functions, i.e., we define $F_{i}^{-}(t, x):=\mathbb{P}(I(t)=-, X(t)=$ $i, W(t) \leq x)$ and $F_{i}^{+}(t, x)$ analogously. 
$1^{+}: F_{i}^{+}(x)$ in Regime 1 . Regime 1 refers to $0<x<B_{2}$, where we have for small $h$

$$
\begin{aligned}
F_{i}^{+}(t+h, x)= & \left(1-h \sum_{j \neq i} q_{i, j}^{+}\right) F_{i}^{+}\left(t, x-h\left(r_{i}^{+}-c\right)\right) \\
& +h \sum_{j \neq i} q_{j, i}^{+} F_{j}^{+}(t, x)+o(h) .
\end{aligned}
$$

Rearranging, dividing by $h$, and using the fact that the rows of the $Q^{+}$matrix add up to zero, we obtain

$$
\begin{aligned}
& \frac{F_{i}^{+}(t+h, x)-F_{i}^{+}\left(t, x-h\left(r_{i}^{+}-c\right)\right)}{h} \\
& \quad=q_{i, i}^{+} F_{i}^{+}\left(t, x-h\left(r_{i}^{+}-c\right)\right)+\sum_{j \neq i} q_{j, i}^{+} F_{j}^{+}(t, x)+\frac{o(h)}{h} .
\end{aligned}
$$

By taking $h \downarrow 0$, we find

$$
\frac{\partial}{\partial t} F_{i}^{+}(t, x)+\left(r_{i}^{+}-c\right) \frac{\partial}{\partial x} F_{i}^{+}(t, x)=\sum_{j} q_{j, i}^{+} F_{j}^{+}(t, x) .
$$

(Remark that, formally, these partial derivatives are not necessarily well-defined. As we are interested in the stationary behavior of the queue, this fact does not play a role - in fact we can assume the queue content has a proper density at time 0.) Assuming stationarity we set $F_{i}^{+}(t, x)=F_{i}^{+}(x)$ and in addition we set all derivatives with respect to $t$ equal to 0 . We thus obtain

$$
\left(r_{i}^{+}-c\right) \frac{\mathrm{d}}{\mathrm{d} x} F_{i}^{+}(x)=\sum_{j} q_{j, i}^{+} F_{j}^{+}(x) .
$$

$2^{+}: F_{i}^{+}(x)$ in Regime 2. We now consider the interval $B_{2}<x<B_{1}$. For $i$ in $S_{\mathrm{U}}^{-}$, we simply have the same equations as in Regime 1, leading to Eq. (4). However, when $i$ in $S_{\mathrm{D}}^{-}$, we have to include the possibility that a transition can occur from the '-' state into the ' + ' state. This will happen when the buffer content at time $t$ is between $B_{2}$ and $B_{2}-h\left(r_{i}^{-}-c\right)$ (which is just above $B_{2}$ due to $i \in S_{\mathrm{D}}^{-}$), and the background process does not change its state during $(t, t+h]$. A complication here is that before the transition, $Q^{-}$is active and after the transition $Q^{+}$is active. However, we do know that the probability that $X(t)$ remains in $i$ during during $(t, t+h]$ is $1+o(1)$, no matter what the precise form is, and this knowledge is sufficient. We thus find, for $i$ in $S_{\mathrm{D}}^{-}$, 


$$
\begin{aligned}
F_{i}^{+}(t+h, x)= & \left(1-h \sum_{j \neq i} q_{i, j}^{+}\right) F_{i}^{+}\left(t, x-h\left(r_{i}^{+}-c\right)\right)+h \sum_{j \neq i} q_{j, i}^{+} F_{j}^{+}(t, x) \\
& +(1+o(1))\left(F_{i}^{-}\left(t, B_{2}-h\left(r_{i}^{-}-c\right)\right)-F_{i}^{-}\left(t, B_{2}\right)\right)+o(h) .
\end{aligned}
$$

By rearranging and dividing by $h$ on both sides we obtain

$$
\begin{aligned}
& \frac{F_{i}^{+}(t+h, x)-F_{i}^{+}\left(t, x-h\left(r_{i}^{+}-c\right)\right)}{h} \\
& =q_{i, i}^{+} F_{i}^{+}\left(t, x-h\left(r_{i}^{+}-c\right)\right)+\sum_{j \neq i} q_{j, i}^{+} F_{j}^{+}(t, x)+(1+o(1)) \\
& \quad \times \frac{\left(F_{i}^{-}\left(t, B_{2}-h\left(r_{i}^{-}-c\right)\right)-F_{i}^{-}\left(t, B_{2}\right)\right.}{h}+\frac{o(h)}{h} .
\end{aligned}
$$

Then taking $h \downarrow 0$, and assuming stationarity we find

$$
\left(r_{i}^{+}-c\right) \frac{\mathrm{d}}{\mathrm{d} x} F_{i}^{+}(x)=\sum_{j} q_{j, i}^{+} F_{j}^{+}(x)-\left.\left(r_{i}^{-}-c\right) \frac{\mathrm{d}}{\mathrm{d} x} F_{i}^{-}(x)\right|_{x \downarrow B_{2}} .
$$

Since for $i$ in $S_{\mathrm{U}}^{-}$, we already found the same equations as in Regime 1, leading to Eq. (4), we can combine the two cases to find, for $i=1, \ldots, d$,

$$
\left(r_{i}^{+}-c\right) \frac{\mathrm{d}}{\mathrm{d} x} F_{i}^{+}(x)=\sum_{j} q_{j, i}^{+} F_{j}^{+}(x)-A_{i}^{-},
$$

where

$$
A_{i}^{-}:= \begin{cases}\left.\left(r_{i}^{-}-c\right) \frac{\mathrm{d}}{\mathrm{d} x} F_{i}^{-}(x)\right|_{x \downarrow B_{2}} & \text { for } i \text { in } S_{\mathrm{D}}^{-} \\ 0 & \text { for } i \text { in } S_{\mathrm{U}}^{-}\end{cases}
$$

$2^{-}: F_{i}^{-}(x)$ in Regime 2. In Regime 2, i.e., $B_{2}<x<B_{1}$, for $i$ in $S_{\mathrm{U}}^{-}$, we have the simple case

$$
\begin{aligned}
F_{i}^{-}(t+h, x)= & \left(1-h \sum_{j \neq i} q_{i, j}^{-}\right) F_{i}^{-}\left(t, x-h\left(r_{i}^{-}-c\right)\right) \\
& +h \sum_{j \neq i} q_{j, i}^{-} F_{j}^{-}(t, x)+o(h) .
\end{aligned}
$$


By rearranging, dividing by $h$, taking $h \downarrow 0$ and assuming stationarity we obtain

$$
\left(r_{i}^{-}-c\right) \frac{\mathrm{d}}{\mathrm{d} x} F_{i}^{-}(x)=\sum_{j} q_{j, i}^{-} F_{j}^{-}(x) .
$$

For $i \in S_{\mathrm{D}}^{-}$, the equation is more complicated. If we consider a time interval of $h$ time units, then in this interval the buffer occupancy will drop by $\left|h\left(r_{i}^{-}-c\right)\right|$. We have to make sure that it does not drop to or below $B_{2}$. If this occurs then the indicator switches to the ' + ' state, which is the probability we want to subtract from the equations [as it was already taken into account in (6)]. Therefore, we include the term $-F_{i}^{-}\left(t, B_{2}-h\left(r_{i}^{-}-c\right)\right)$ which ensures that after $h$ time units the buffer occupancy cannot drop to or below $B_{2}$. We thus obtain

$$
\begin{aligned}
F_{i}^{-}(t+h, x)= & \left(1-h \sum_{j \neq i} q_{i, j}^{-}\right)\left(F_{i}^{-}\left(t, x-h\left(r_{i}^{-}-c\right)\right)\right. \\
& \left.-F_{i}^{-}\left(t, B_{2}-h\left(r_{i}^{-}-c\right)\right)\right)+h \sum_{j \neq i} q_{j, i}^{-} F_{j}^{-}(t, x)+o(h) .
\end{aligned}
$$

By rearranging, taking $h \downarrow 0$ and assuming stationarity we get

$$
\left(r_{i}^{-}-c\right) \frac{\mathrm{d}}{\mathrm{d} x} F_{i}^{-}(t, x)=\sum_{j} q_{j, i}^{-} F_{j}^{-}(t, x)+\left.\left(r_{i}^{-}-c\right) \frac{\mathrm{d}}{\mathrm{d} x} F_{i}^{-}(t, x)\right|_{x \downarrow B_{2}} .
$$

We can now combine Eqs. (9) and (10) into one equation for $i$ as

$$
\left(r_{i}^{-}-c\right) \frac{\mathrm{d}}{\mathrm{d} x} F_{i}^{-}(x)=\sum_{j} q_{j, i}^{-} F_{j}^{-}(x)+A_{i}^{-}
$$

where $A_{i}^{-}$is given by Eq. (8).

$3^{-}: F_{i}^{-}(x)$ in Regime 3. The equations for $B_{1}<x<\infty$, are the most complicated. This is because we have to take into account two aspects. First, we have to exclude the possibility of a transition from the ' - ' into the ' + ' state when $X(t)$ is in $S_{\mathrm{D}}^{-}$and the buffer content is just above $B_{2}$ at time $t$. It is clear from the explanation for $F_{i}^{-}(x)$ in Regime 2 that this is done by including a term $-F_{i}^{-}\left(t, B_{2}-h\left(r_{i}^{-}-c\right)\right)$. Second, we have to include the possibility of a transition from the ' + ' state into the ' - ' state. If at time $t$, the buffer content is somewhere in the interval $\left[B_{1}, B_{1}-h\left(r_{i}^{+}-c\right)\right]$, and the background state is in $S_{\mathrm{U}}^{+}$, then in another $h$ time units, the buffer content will increase and will definitely reach $B_{1}$ and jump into the '-' state. We therefore add a term $(1+o(1))\left(F_{i}^{+}\left(t, B_{1}\right)-F_{i}^{+}\left(t, B_{1}-h\left(r_{i}^{+}-c\right)\right)\right)$, similar to the term $(1+o(1))\left(F_{i}^{-}\left(t, B_{2}-h\left(r_{i}^{-}-c\right)\right)-F_{i}^{-}\left(t, B_{2}\right)\right)$ we added in the equation for $F_{i}^{+}$in Regime 2. We find, for $i$ in $S_{\mathrm{D}}^{-} \cap S_{\mathrm{U}}^{+}$, 


$$
\begin{aligned}
F_{i}^{-}(t+h, x)= & \left(1-h \sum_{j \neq i} q_{i, j}^{-}\right) \\
& \times\left(F_{i}^{-}\left(t, x-h\left(r_{i}^{-}-c\right)\right)-F_{i}^{-}\left(t, B_{2}-h\left(r_{i}^{-}-c\right)\right)\right) \\
& +(1+o(1))\left(F_{i}^{+}\left(t, B_{1}\right)-F_{i}^{+}\left(t, B_{1}-h\left(r_{i}^{+}-c\right)\right)\right) \\
& +h \sum_{j \neq i} q_{j, i}^{-} F_{j}^{-}(t, x)+o(h) .
\end{aligned}
$$

By rearranging, dividing by $h$, taking $h \downarrow 0$, and assuming stationarity, we obtain

$$
\begin{aligned}
\left(r_{i}^{-}-c\right) \frac{\mathrm{d}}{\mathrm{d} x} F_{i}^{-}(x)= & \sum_{j} q_{j, i}^{-} F_{j}^{-}(x)+\left.\left(r_{i}^{-}-c\right) \frac{\mathrm{d}}{\mathrm{d} x} F_{i}^{-}(x)\right|_{x \downarrow B_{2}} \\
& +\left.\left(r_{i}^{+}-c\right) \frac{\mathrm{d}}{\mathrm{d} x} F_{i}^{+}(x)\right|_{x \uparrow B_{1}}
\end{aligned}
$$

In order to derive the equations for the other values of $i$ we should note that in Eq. (12) the term $F_{i}^{-}\left(t, B_{2}-h\left(r_{i}^{-}-c\right)\right)$ appears for all $i \in S_{\mathrm{D}}^{-}$and the term $(1+o(1))\left(F_{i}^{+}\left(t, B_{1}\right)-F_{i}^{+}\left(t, B_{1}-h\left(r_{i}^{+}-c\right)\right)\right)$ for $i \in S_{\mathrm{U}}^{+}$. Further on in (13) the term $F_{i}^{-}\left(t, B_{2}-h\left(r_{i}^{-}-c\right)\right)$ results in

$$
\left.\left(r_{i}^{-}-c\right) \frac{\mathrm{d}}{\mathrm{d} x} F_{i}^{-}(x)\right|_{x \downarrow B_{2}},
$$

whereas $(1+o(1))\left(F_{i}^{+}\left(t, B_{1}\right)-F_{i}^{+}\left(t, B_{1}-h\left(r_{i}^{+}-c\right)\right)\right)$ leads to

$$
\left.\left(r_{i}^{+}-c\right) \frac{\mathrm{d}}{\mathrm{d} x} F_{i}^{+}(x)\right|_{x \uparrow B_{1}}
$$

Therefore, we obtain, for any $i=1, \ldots, d$,

$$
\left(r_{i}^{-}-c\right) \frac{\mathrm{d}}{\mathrm{d} x} F_{i}^{-}(x)=\sum_{j} q_{j, i}^{-} F_{j}^{-}(x)+A_{i}^{-}+A_{i}^{+},
$$

where $A_{i}^{-}$is given by Eq. (8) and $A_{i}^{+}$is

$$
A_{i}^{+}:= \begin{cases}\left.\left(r_{i}^{+}-c\right) \frac{\mathrm{d}}{\mathrm{d} x} F_{i}^{+}(x)\right|_{x \uparrow B_{1}} & \text { for } i \in S_{\mathrm{U}}^{+} \\ 0 & \text { for } i \in S_{\mathrm{D}}^{+}\end{cases}
$$


Table 1 Overview of balance equations in the different regimes

\begin{tabular}{llll}
\hline Regime & Interval & $\boldsymbol{F}^{+}(x)$ & $\boldsymbol{F}^{-}(x)$ \\
\hline 1 & $\left(0, B_{2}\right)$ & $\frac{\mathrm{d}}{\mathrm{d} x} \boldsymbol{F}^{+}(x)\left(R^{+}-C\right)=\boldsymbol{F}^{+}(x) Q^{+}$ & 0 \\
2 & $\left(B_{2}, B_{1}\right)$ & $\frac{\mathrm{d}}{\mathrm{d} x} \boldsymbol{F}^{+}(x)\left(R^{+}-C\right)=\boldsymbol{F}^{+}(x) Q^{+}-\boldsymbol{A}^{-}$ & $\frac{\mathrm{d}}{\mathrm{d} x} \boldsymbol{F}^{-}(x)\left(R^{-}-C\right)=\boldsymbol{F}^{-}(x) Q^{-}+\boldsymbol{A}^{-}$ \\
3 & $\left(B_{1}, \infty\right)$ & $\boldsymbol{F}^{+}\left(B_{1}\right)$ & $\frac{\mathrm{d}}{\mathrm{d} x} \boldsymbol{F}^{-}(x)\left(R^{-}-C\right)=\boldsymbol{F}^{-}(x) Q^{-}+\boldsymbol{A}^{-}+\boldsymbol{A}^{+}$ \\
\hline
\end{tabular}

In order to get an overview of the balance equations in the different regimes we have summarized the results so far in matrix form in Table 1 , where $\boldsymbol{F}^{+}(x) \equiv$ $\left(F_{1}^{+}(x), \ldots, F_{d}^{+}(x)\right)$; the row vectors $\boldsymbol{F}^{-}(x), \boldsymbol{A}^{-}$and $\boldsymbol{A}^{+}$are defined similarly. $R^{+}$is the diagonal matrix $\operatorname{diag}\left\{r_{1}^{+}, \ldots, r_{d}^{+}\right\}$and $R^{-}$the diagonal matrix $\operatorname{diag}\left\{r_{1}^{-}, \ldots, r_{d}^{-}\right\}$. We also introduce $C:=c I_{d}$, where $I_{d}$ is the identity matrix of dimension $d$.

\subsection{Solution to the balance equations}

In the previous subsection we have derived the balance equations for both $\boldsymbol{F}^{-}(x)$ and $\boldsymbol{F}^{+}(x)$. In this subsection we provide the solutions to these equations, using the spectral expansion method used in Anick et al. (1982) and Mandjes et al. (2003). The solution can then be presented in a simple form, but it involves a number of unknown coefficients.

$1^{+}$: The balance equation for $\boldsymbol{F}^{+}(x)$ in Regime 1 is $\mathrm{d} \boldsymbol{F}^{+}(x) / \mathrm{d} x \cdot\left(R^{+}-C\right)=$ $F^{+}(x) Q^{+}$. The spectral expansion of the solution to this equation is given by

$$
\boldsymbol{F}^{+}(x)=\sum_{j=1}^{d} a_{j}^{1+} \boldsymbol{v}_{j}^{+} \exp \left[z_{j}^{+} x\right],
$$

where $\left(z_{j}^{+}, \boldsymbol{v}_{j}^{+}\right)$is an eigenvalue-eigenvector pair statisfying $z_{j}^{+} \boldsymbol{v}_{j}^{+}\left(R^{+}-C\right)=$ $\boldsymbol{v}_{j}^{+} Q^{+}$, and the $a_{j}^{1+}$ are coefficients.

In the above solution we tacitly assumed that the matrix $Q^{+}\left(R^{+}-C\right)^{-1}$ has full eigenspace, in that all eigenvalues are simple (i.e., have multiplicity 1 ). Two remarks are in place here. (A) In the first place, we mention that it is known that if the $Q^{+}$matrix has a specific structure, the eigenvalues are indeed simple (and real); most notably, as shown in van Doorn et al. (1988), if $Q^{+}$corresponds to a birth-death Markov process this property indeed applies. (B) Secondly, eigenvalues with multiplicity $k$ larger than 1 do not lead to any conceptual problems. Standard theory on linear differential equations entails that then the density of the stationary queue content has terms proportional to $x^{j} \exp \left(-z_{j}^{+} x\right)$, with $j=0, \ldots, k-1$. We decided to assume in our analysis that the eigenvalues of $Q^{+}\left(R^{+}-C\right)^{-1}$ (and later also those of $Q^{-}\left(R^{-}-C\right)^{-1}$ ) are simple as the corresponding formulas for the 'non-simple case' do not add much extra insight, and are notationally cumbersome. 
$2^{+}$: We now consider $\boldsymbol{F}^{+}(x)$ in the interval $B_{2}<x<B_{1}$ for which the balance equation is

$$
\frac{\mathrm{d}}{\mathrm{d} x} \boldsymbol{F}^{+}(x)\left(R^{+}-C\right)=\boldsymbol{F}^{+}(x) Q^{+}-\boldsymbol{A}^{-} .
$$

This equation has an inhomogeneous term because of which we cannot write the solution as for $\boldsymbol{F}^{+}(x)$ in Regime 1. Since $A_{i}^{-}$is a constant [see Eq. (8)], differentiation of the above equation with respect to $x$ gives us a homogeneous equation in $\boldsymbol{f}^{+}(x) \equiv \mathrm{d} \boldsymbol{F}^{+}(x) / \mathrm{d} x$ as below

$$
\frac{\mathrm{d}}{\mathrm{d} x} f^{+}(x)\left(R^{+}-C\right)=f^{+}(x) Q^{+} .
$$

Now that we have a homogeneous equation its solution is given as

$$
\boldsymbol{f}^{+}(x)=\sum_{j=1}^{d} \tilde{a}_{j}^{2+} \boldsymbol{v}_{j}^{+} \exp \left[z_{j}^{+} x\right]
$$

where $\left(z_{j}^{+}, \boldsymbol{v}_{j}^{+}\right)$is the same eigenvalue-eigenvector pair as before and $\tilde{a}_{j}^{2+}$ are coefficients. As $Q^{+}$is the generator, it has eigenvalue 0 , and hence one of the eigenvalues $z_{j}^{+}$is zero, say $z_{j^{*}}^{+}=0$. With this in mind, integration immediately yields that

$$
\boldsymbol{F}^{+}(x)=\sum_{j=1, j \neq j^{*}}^{d} a_{j}^{2+} \boldsymbol{v}_{j}^{+} \exp \left[z_{j}^{+} x\right]+a_{j^{*}}^{2+} \boldsymbol{v}_{j^{*}}^{+} x+\boldsymbol{w}^{2+},
$$

where $a_{j}^{2+}=\tilde{a}_{j}^{2+} / z_{j}^{+}$for $j \neq j^{*}, a_{j^{*}}^{2+}=\tilde{a}_{j^{*}}^{2+}$, and the components $w_{i}^{2+}$ of $\boldsymbol{w}^{2+}$ are integration constants.

$2^{-}$: For $B_{2}<x<B_{1}$, the balance equation for $\boldsymbol{F}^{-}(x)$ is $\mathrm{d} \boldsymbol{F}^{-}(x) / \mathrm{d} x \cdot\left(R^{-}-C\right)=$ $\boldsymbol{F}^{-}(x) Q^{-}+\boldsymbol{A}^{-}$. This is again a inhomogeneous equation and the spectral method cannot be used directly. Therefore, we follow the same procedure as for $\boldsymbol{F}^{+}(x)$ in Regime 2. We differentiate the equation on both sides to get a homogeneous equation in $f^{-}(x)$, which we integrate to obtain

$$
\boldsymbol{F}^{-}(x)=\sum_{j=1, j \neq j^{*}}^{d} a_{j}^{2-} \boldsymbol{v}_{j}^{-} \exp \left[z_{j}^{-} x\right]+a_{j^{*}}^{2-} \boldsymbol{v}_{j^{\star}}^{-} x+\boldsymbol{w}^{2-}
$$

where $\left(z_{j}^{-}, \boldsymbol{v}_{j}^{-}\right)$is an eigenvalue-eigenvector pair satisfying $z_{j}^{-} \boldsymbol{v}_{j}^{-}\left(R^{-}-C\right)=$ $\boldsymbol{v}_{j}^{-} Q^{-}$, and the $a_{j}^{2-}$ are coefficients. The components $w_{i}^{2-}$ of $\boldsymbol{w}^{2-}$, are integration constants and the coefficient $a_{j^{\star}}^{2-}$ corresponds to the eigenvalue $z_{j^{\star}}^{-}=0$ of $Q^{-}$. 
Table 2 Overview of solution

\begin{tabular}{llll}
\hline Regime & Interval & $\boldsymbol{F}^{+}(x)$ & $\boldsymbol{F}^{-}(x)$ \\
\hline 1 & $\left(0, B_{2}\right)$ & $\sum_{j=1}^{d} a_{j}^{1+} \boldsymbol{v}_{j}^{+} \exp \left[z_{j}^{+} x\right]$ & 0 \\
2 & $\left(B_{2}, B_{1}\right)$ & $\begin{array}{c}\sum_{j=1, j \neq j^{\star}}^{d} a_{j}^{2+} \boldsymbol{v}_{j}^{+} \exp \left[z_{j}^{+} x\right] \\
+a_{j^{\star}}^{2+} \boldsymbol{v}_{j^{\star}}^{+} x+\boldsymbol{w}^{2+}\end{array}$ & $\begin{array}{c}\sum_{j=1, j \neq j^{\star}}^{d} a_{j}^{2-} \boldsymbol{v}_{j}^{-} \exp \left[z_{j}^{-} x\right] \\
+a_{j^{\star}}^{2-} \boldsymbol{v}_{j^{\star}}^{-} x+\boldsymbol{w}^{2-}\end{array}$ \\
& & $F^{+}\left(B_{1}\right)$ & $\begin{array}{c}\sum_{j=1, j \neq j^{\star}}^{d} a_{j}^{3-} \boldsymbol{v}_{j}^{-} \exp \left[z_{j}^{-} x\right] \\
3\end{array}$ \\
& & $\left.+a_{1}^{3-}, \infty\right)$ & \\
& &
\end{tabular}

$3^{-}$: The balance equation for $\boldsymbol{F}^{-}(x)$ in Regime 3 is $\mathrm{d} \boldsymbol{F}^{-}(x) / \mathrm{d} x \cdot\left(R^{-}-C\right)=$ $\boldsymbol{F}^{-}(x) Q^{-}+\boldsymbol{A}^{-}+\boldsymbol{A}^{+}$. In this equation we have two inhomogeneous terms as opposed to one in the previous cases. Nevertheless, we can still apply the same method as for $\boldsymbol{F}^{+}(x)$ in Regime 2 and $\boldsymbol{F}^{-}(x)$ in Regime 2. This is because both the inhomogeneous terms in the equation above consist of constant elements $\lim _{x \downarrow B_{2}} \mathrm{~d} F_{i}^{-}(x) / \mathrm{d} x$ and $\lim _{x \uparrow B_{1}} \mathrm{~d} F_{i}^{+}(x) / \mathrm{d} x$ or zero [see Eqn. (8) and (15)] which disappear after differentiating with respect to $x$. Therefore, after differentiation and then integration we get the solution for $\boldsymbol{F}^{-}(x)$ in Regime 3 as

$$
\boldsymbol{F}^{-}(x)=\sum_{j=1, j \neq j^{\star}}^{d} a_{j}^{3-} \boldsymbol{v}_{j}^{-} \exp \left[z_{j}^{-} x\right]+a_{j^{\star}}^{3-} \boldsymbol{v}_{j^{\star}}^{-} x+\boldsymbol{w}^{3-},
$$

where $\left(z_{j}^{-}, \boldsymbol{v}_{j}^{-}\right)$is again the eigenvalue-eigenvector pair that satisfies $z_{j}^{-} \boldsymbol{v}_{j}^{-}\left(R^{-}-C\right)=\boldsymbol{v}_{j}^{-} Q^{-}$, and the $a_{j}^{3-}$ are coefficients. The components $w_{i}^{3-}$ of $\boldsymbol{w}^{3-}$ are integration constants and the coefficient $a_{j^{\star}}^{3-}$ corresponds to $z_{j^{\star}}^{-}=0$.

We summarize the solutions found in the various intervals in Table 2.

\subsection{Derivation of conditions for finding the unknowns in the solution}

In Sect. 3.2, we provided the solution to $\boldsymbol{F}^{-}(x)$ and $\boldsymbol{F}^{+}(x)$ using the spectral expansion method. However, the solution includes many unknowns which need to be found with additional conditions. Table 2 presents an overview of the solution where the vectors $\boldsymbol{a}^{1+}, \boldsymbol{a}^{2+}, \boldsymbol{a}^{2-}, \boldsymbol{a}^{3-}, \boldsymbol{w}^{2+}, \boldsymbol{w}^{2-}$ and $\boldsymbol{w}^{3-}$ are unknown. Table 3 enumerates all unknowns giving a total of $8 d$. In this section our goal is to find $8 d$ conditions so as to solve the system.

A. Boundary conditions at $x=0$ and $x=\infty$ are as in Anick et al. (1982) and Kosten (1984):

- $F_{i}^{+}(0)=0$, for $i$ in $S_{\mathrm{U}}^{+}$. This is because it cannot be that simultaneously the buffer is empty and the background process is in an up-state. This gives us $d_{\mathrm{U}}^{+}$ conditions. 
Table 3 Overview of the unknowns in the different regimes

\begin{tabular}{lllll}
\hline Regime & Interval & $\boldsymbol{F}^{+}(x)$ & $\boldsymbol{F}^{-}(x)$ & \\
\hline 1 & $\left(0, B_{2}\right)$ & $a_{j}^{1+} \quad j=1, \ldots, d$ & 0 & \\
2 & $\left(B_{2}, B_{1}\right)$ & $a_{j}^{2+}, w_{j}^{2+} \quad j=1, \ldots, d$ & $a_{j}^{2-}, w_{j}^{2-}$ & $j=1, \ldots, d$ \\
3 & $\left(B_{1}, \infty\right)$ & $F_{j}^{+}\left(B_{1}\right)$, for $j=1, \ldots, d$ & $a_{j}^{3-}, w_{j}^{3-}$ & $j=1, \ldots, d$ \\
& & Total number of unknowns: $8 d$ & & \\
\hline
\end{tabular}

- For $x \rightarrow \infty, F_{i}^{-}(x)$ should remain bounded, and therefore for all $z_{j}^{-}$with a non-negative real part, the corresponding $a_{j}^{3-}$ has to be zero. Notice that this also entails that the equilibrium distribution of $W^{-}(t)$ is given by $\boldsymbol{w}^{3-}$. This gives us $d_{\mathrm{D}}^{-}$conditions.

B. Continuity conditions. $F_{i}^{+}(x)$ and $F_{i}^{-}(x)$ are both continuous at the thresholds $B_{1}$ and $B_{2}$. This gives us the following $4 d$ equations:

- $\lim _{x \uparrow B_{2}} F_{i}^{+}(x)=\lim _{x \downarrow B_{2}} F_{i}^{+}(x)$.

- $\lim _{x \uparrow B_{1}} F_{i}^{+}(x)=F_{i}^{+}\left(B_{1}\right)$.

- $\lim _{x \downarrow B_{2}} F_{i}^{-}(x)=0$.

- $\lim _{x \downarrow B_{1}} F_{i}^{-}(x)=\lim _{x \downarrow B_{1}} F_{i}^{-}(x)$.

C. Substitution conditions.

As in Mandjes et al. (2003) we need to substitute the solutions given in Sect. 3.2 into the inhomogeneous balance equations of Sect. 3.1. We have 3 inhomogeneous systems. Potentially each of these can lead to $d$ conditions. Therefore, in total we would get $3 d$ equations from the substitution.

Boundary conditions, continuity conditions and substitution conditions together were sufficient to solve the model in Mandjes et al. (2003), but as we have a more complicated model in which the fluid source alternates between two modes, this is not the case here. Therefore, we introduce and prove the following:

D. Additional conditions.

In the first place, suppose that the buffer is filling up in the ' + ' state. At some point it will reach $B_{1}$ and then switch to the '-' process. Since there is no density beyond $B_{1}$ (and the phase being ' + ') it is highly unlikely that the buffer level is just below $B_{1}$ while the background process is in a down-state. Similarly, when the buffer is filling up ('-' phase) it is unlikely that the buffer content is just above $B_{2}$ while the background process is in an up-state.

The lemma below states these additional conditions more precisely, and is proved by deriving the balance equations at $x=B_{1}$ and $x=B_{2}$. Note that these were not addressed in Sect. 3.1.

Lemma 3.1 (i) For all $i \in S_{\mathrm{D}}^{+}$,

$$
\left.\frac{\mathrm{d}}{\mathrm{d} x} F_{i}^{+}(x)\right|_{x \uparrow B_{1}}=0 .
$$


(ii) For all $i \in S_{\mathrm{U}}^{-}$

$$
\left.\frac{\mathrm{d}}{\mathrm{d} x} F_{i}^{-}(x)\right|_{x \downarrow B_{2}}=0
$$

Proof (i) We first consider the case for $i$ in $S_{\mathrm{D}}^{-} \cap S_{\mathrm{U}}^{+}$. In this case we have

$$
\begin{aligned}
F_{i}^{+}\left(t+h, B_{1}\right)= & \left(1-h \sum_{j \neq i} q_{i, j}^{+}\right) F_{i}^{+}\left(t, B_{1}-h\left(r_{i}^{+}-c\right)\right)+h \sum_{j \neq i} q_{j, i}^{+} F_{j}^{+}\left(t, B_{1}\right) \\
& +(1+o(1))\left(F_{i}^{-}\left(t, B_{2}-h\left(r_{i}^{-}-c\right)\right)-F_{i}^{-}\left(t, B_{2}\right)\right)+o(h) .
\end{aligned}
$$

By rearranging, dividing by $h$, taking $h \downarrow 0$ and assuming stationarity we obtain for $i$ in $S_{\mathrm{D}}^{-} \cap S_{\mathrm{U}}^{+}$,

$$
\left.\left(r_{i}^{+}-c\right) \frac{\mathrm{d}}{\mathrm{d} x} F_{i}^{+}(x)\right|_{x \uparrow B_{1}}=\sum_{j} q_{j, i}^{+}\left(F_{j}^{+}\left(B_{1}\right)\right)-\left.\left(r_{i}^{-}-c\right) \frac{\mathrm{d}}{\mathrm{d} x} F_{i}^{-}(x)\right|_{x \downarrow B_{2}} .
$$

Comparison with (8) and (15) shows that

$$
\sum_{j} q_{j, i}^{+} F_{j}^{+}\left(B_{1}\right)=A_{i}^{+}+A_{i}^{-}
$$

and it is in fact easy to see that this holds for any $i$. Let us compare this to Eq. (7) for $F_{j}^{+}(x)$ in the interval $\left(B_{2}, B_{1}\right)$, letting $x \uparrow B_{1}$, which gives

$$
\sum_{j} q_{j, i}^{+} F_{j}^{+}\left(B_{1}\right)=\left.\left(r_{i}^{+}-c\right) \frac{\mathrm{d}}{\mathrm{d} x} F_{i}^{+}(x)\right|_{x \uparrow B_{1}}+A_{i}^{-} .
$$

From this comparison we conclude that for $i$ in $S_{\mathrm{D}}^{+}$

$$
\left.\left(r_{i}^{+}-c\right) \frac{\mathrm{d}}{\mathrm{d} x} F_{i}^{+}(x)\right|_{x \uparrow B_{1}}=A_{i}^{+}=0 .
$$

from which the first claim immediately follows.

(ii) The proof of this part is similar, comparing the two equations for $F_{i}^{-}(x)$ at $B_{2}$, which are $F_{i}^{-}\left(B_{2}\right)=0$ and

$$
\left.\left(r_{i}^{-}-c\right) \frac{\mathrm{d}}{\mathrm{d} x} F_{i}^{-}(x)\right|_{x \downarrow B_{2}}=\sum_{j} q_{j, i}^{+} F_{j}^{-}\left(B_{2}\right)+A_{i}^{-} .
$$


The following lemma entails that the number of substitution conditions is really only $2 d$ (not $3 d$ ).

Lemma 3.2 The equations from the substitution condition for $\boldsymbol{F}^{-}(x)$ in the interval $\left(B_{2}, B_{1}\right)$ are implied by the continuity condition for $\boldsymbol{F}^{-}(x)$ at $B_{2}$.

Proof We start with the substitution for $F_{j}^{-}(x)$ in the interval $\left(B_{2}, B_{1}\right)$. The balance equation in this case is, see (11),

$$
\left(r_{i}^{-}-c\right) \frac{\mathrm{d}}{\mathrm{d} x} F_{i}^{-}(x)=\sum_{k} q_{k, i}^{-} F_{k}^{-}(x)+A_{i}^{-} .
$$

On substituting the solution back into the equation and using the fact that $z_{j^{\star}}^{-}=0$ and $\sum_{k} q_{k, i}^{-} v_{j, k}^{-}=z_{j}^{-} v_{j, i}^{-}\left(r_{i}^{-}-c\right)$, where $v_{j, i}^{-}$refers to the $i$ th component of vector $v_{j}^{-}$, we find

$$
\left(r_{i}^{-}-c\right)\left(a_{j^{\star}}^{2-} v_{j^{\star}, i}^{-}\right)=\sum_{j} q_{k, i}^{-} w_{k}^{2-}+A_{i}^{-} .
$$

By the definition of $A_{i}^{-}$and using the first part of Lemma 3.1 we can simply substitute

$$
\begin{aligned}
A_{i}^{-} & =\left.\left(r_{i}^{-}-c\right) \frac{\mathrm{d}}{\mathrm{d} x} F_{i}^{-}(x)\right|_{x \downarrow B_{2}} \\
& =\left(r_{i}^{-}-c\right)\left(\sum_{j=1, j \neq j^{\star}}^{d} a_{j}^{2-} v_{j, i}^{-} z_{j}^{-} \exp \left[z_{j}^{-} B_{2}\right]+a_{j^{\star}}^{2-} v_{j^{\star}, i}^{-}\right)
\end{aligned}
$$

for all $i=1, \ldots, d$ in the equation above to give us

$$
\begin{aligned}
& \sum_{k} q_{k, i}^{-} w_{k}^{2-}+\left(r_{i}^{-}-c\right)\left(\sum_{j=1, j \neq j^{\star}}^{d} a_{j}^{2-} v_{j, i}^{-} z_{j}^{-} \exp \left[z_{j}^{-} B_{2}\right]\right)=0, \quad \text { or }, \\
& \sum_{k} q_{k, i}^{-} w_{k}^{2-}+\sum_{k} q_{k, i}^{-}\left(\sum_{j=1, j \neq j^{\star}}^{d} a_{j}^{2-} v_{j, k}^{-} \exp \left[z_{j}^{-} B_{2}\right]\right)=0
\end{aligned}
$$

as the substitution conditions should hold. However, since we may freely add $\sum_{k} q_{k, i}^{-} a_{j^{\star}}^{2-} v_{j^{\star}, k}^{-} B_{2}$ to the left-hand side of the above (since it is zero), and then combine the sums into one, the conditions turn out to be equivalent to $\sum_{k} q_{k, i}^{-} F_{k}^{-}\left(B_{2}\right)=0$, Hence they are indeed implied by the continuity equations at $B_{2}$, which say that $F_{k}^{-}\left(B_{2}\right)=0$ for all $k$.

Let us now explain the intuition behind Lemma 3.2. If we look at Table 1, we could suspect that an overlap could arise for $F_{i}^{-}(x)$ in Regime 2. This is because this is the only inhomogeneous equation which involves a single indicator state (being the '-' state). The continuity equations would also have the same characteristics. As for the other inhomogeneous equations, these involve terms with both the ' + ' and the 
'-' states, whereas the continuity equations still involve terms with a single indicator state. Hence, for these cases there is a clear difference between the characteristics of the substitution and the continuity equations, and the substitution equations do give additional information.

Let us now count the number of conditions we have at our disposal. The boundary conditions give us $d_{\mathrm{U}}^{+}$and $d_{\mathrm{D}}^{-}$equations and the four continuity conditions give us another $4 d$ conditions. The substitution conditions could have potentially given us another $3 d$ conditions. Adding these to the $d_{\mathrm{D}}^{+}+d_{\mathrm{U}}^{-}$conditions from Lemma 3.1, we would together have $9 d$ conditions, $d$ more than we need. With Lemma 3.2 we proved that an overlap of $d$ conditions exists between the continuity and the substitution conditions, eventually adding up to exactly $8 d$ conditions equal to the total number of unknowns in the solution (see Table 3), which we need to solve the system.

However, it is important to note that all the $8 d$ equations are linear in the different unknowns enlisted in Table 3, with a rank of $8 d-1$. This is easy to see since the linear system can be solved upto a multiplicative constant. The redundancy in the equations can be removed by replacing any one of the equations in the linear system by the normalization equation

$$
\sum_{i=1}^{d}\left(F_{i}^{+}(\infty)+F_{i}^{-}(\infty)\right)=1
$$

Thus, we arrive at the following result.

Proposition 3.3 We have $8 d$ conditions on the coefficients, matching the number of unknowns.

In the next section we illustrate, by means of a numerical example, how one can identify the $8 d$ unknowns.

\section{Numerical example}

In this section we provide numerical results aimed at demonstrating the computation of the stationary distribution of the buffer content and the other performance measures. We consider a two state numerical example, i.e., with $d=2$. We consider the following generator matrices and rate vectors,

$$
Q^{+}=\left(\begin{array}{cc}
-1 & 1 \\
2 & -2
\end{array}\right), \quad Q^{-}=\left(\begin{array}{cc}
-0.8 & 0.8 \\
5 & -5
\end{array}\right), \quad \boldsymbol{r}^{-}=\left(\begin{array}{c}
16 \\
0
\end{array}\right), \quad \boldsymbol{r}^{+}=\left(\begin{array}{c}
25 \\
0
\end{array}\right)
$$

The other parameters are $c=15, B_{1}=15$ and $B_{2}=10$. The diagonal matrices $R^{-}$, $R^{+}$, and $C$ then equal $\operatorname{diag}\{16,0\}, \operatorname{diag}\{25,0\}$, and $\operatorname{diag}\{15,15\}$, respectively. After the numerical determination of the eigensystems of the matrices $Q^{+}\left(R^{+}-C\right)^{-1}$ and $Q^{-}\left(R^{-}-C\right)^{-1}$ we apply the conditions as listed in Sect. 3.3. We then solve the resulting linear system of equations for the $8 d=16$ unknowns. This gives us the complete and unique solution for the stationary distribution of the buffer content, $\boldsymbol{F}^{+}(x)$ and $\boldsymbol{F}^{-}(x)$. The graphical representation of $F_{i}^{+}(x)$ and $F_{i}^{-}(x)$ for $i=1,2$ is shown in Fig. 2. 

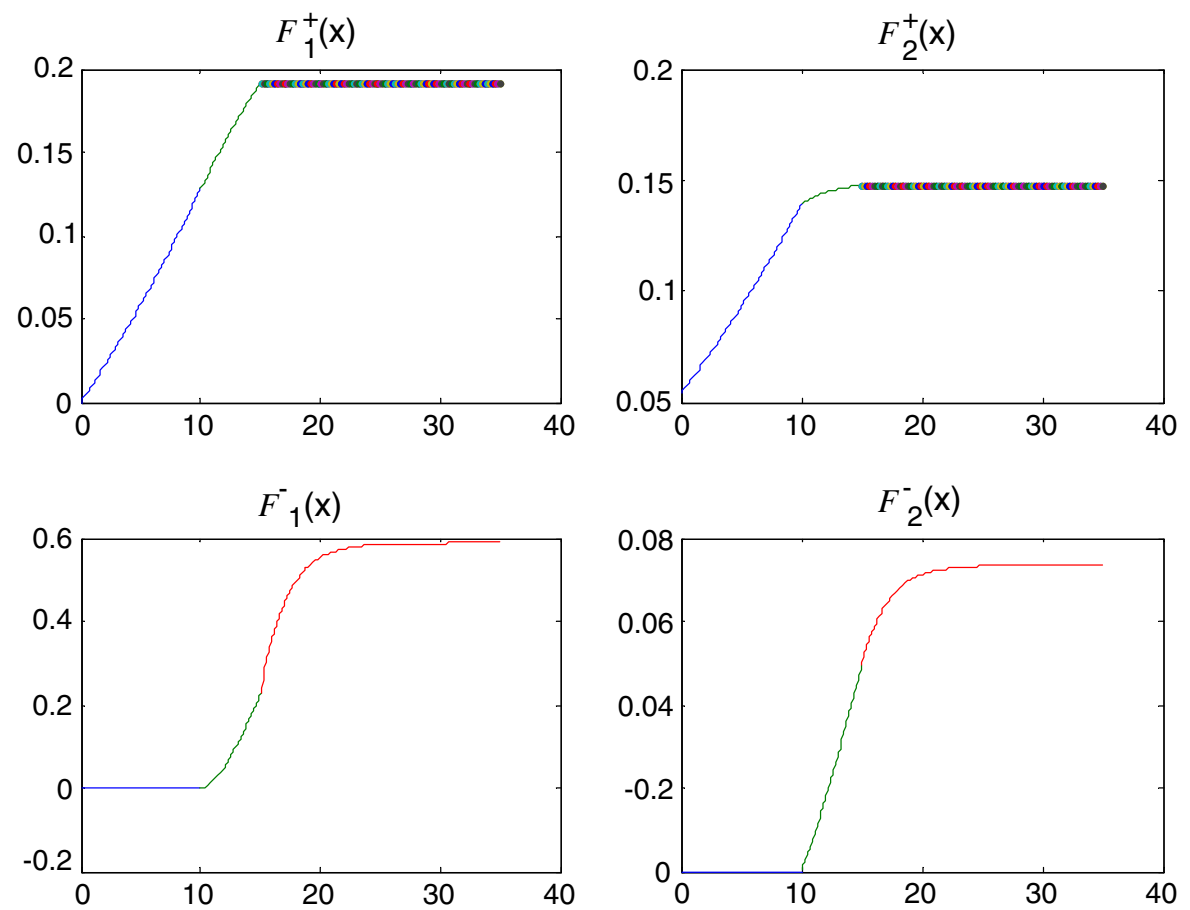

Fig. 2 Probability distribution functions of the buffer content with $d=2, B_{1}=15, B_{2}=10$

The total throughput of the system can be calculated from Eq. (1) as

$$
\vartheta=r_{1}^{+} F_{1}^{+}(\infty)+r_{1}^{-} F_{1}^{-}(\infty)=14.1924
$$

We can compute the expected delay by first computing $F(x)=\sum_{i, j} F_{i}^{j}(x)$, then computing the combined probability density as $f(x)=\mathrm{d} F(x) / \mathrm{d} x$. The expected delay is then given by

$$
\mathbb{E} D=\int_{0}^{\infty} x f(x) \mathrm{d} x=12.2040
$$

In a second experiment we consider the effect of having two thresholds on the signaling overhead. The expected number of phase-transitions per unit time equals

$$
\sum_{i \in S_{\mathrm{U}}^{+}} f_{i}^{+}\left(B_{1}\right)\left(r_{i}^{+}-c\right)+\sum_{i \in S_{\mathrm{D}}^{-}} f_{i}^{-}\left(B_{2}\right)\left(c-r_{i}^{-}\right)
$$

this (plausible) formula is derived in Malhotra et al. (2008). The effect of varying $B_{2}$, for a given value of $B_{1}$, is shown in Fig. 3. The other parameters are as above. We observe that indeed the signaling frequency is reduced by choosing $B_{2}$ smaller than $B_{1}$. 


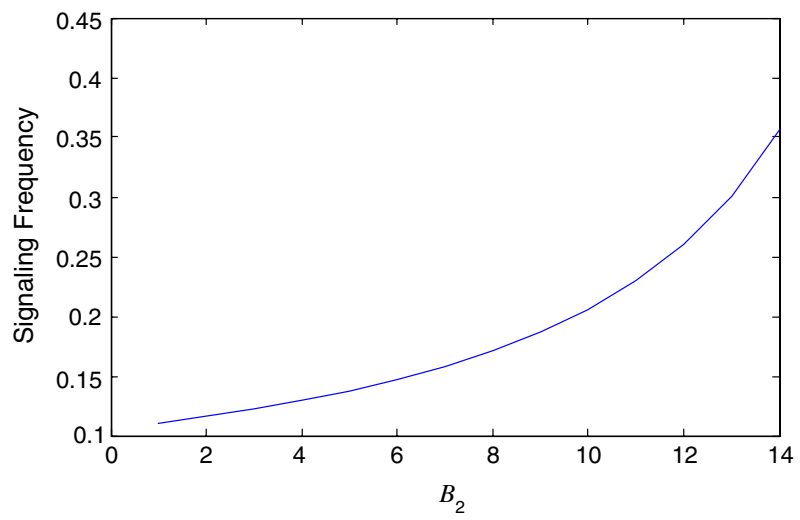

Fig. 3 Signaling frequency as $B_{2}$ grows to $B_{1}=15$

\section{Concluding remarks}

We have analyzed a feedback fluid queueing system in which the traffic source rates adapt to congestion. The system has two thresholds: the higher threshold $B_{1}$ aims at signaling the beginning of a congestion period, whereas the lower threshold $B_{2}$ serves to signal the end of the congestion period. This idea is modelled by letting the input process alternate between two Markov fluid processes: the first applies as long as the upper threshold $B_{1}$ has not been hit from below. As soon as that happens, the traffic source switches to the other process, until $B_{2}$ is hit from above.

The resulting model falls in the class of Markovian feedback fluid queues. The numerical complexity of the methodology used to solve for the buffer content distribution and throughput boils down to solving two $d$-dimensional eigensystems, as well as a (fairly standard) linear system of $8 d$ equations; here $d$ denotes the cardinality of the state space on which the two Markov processes are defined.

A central design problem which can be investigated using our model, and is addressed in Malhotra et al. (2008), is the optimization of the threshold positions while considering the trade-off between throughput and delay. Challenging extensions to the present analysis are the systematic assessment of the signaling frequency in the model (that is, what is the rate at which the input process alternates between the two Markov fluid processes), as a function of the model parameters; this is a relevant issue, as the signaling overhead needs to be controlled. Yet another important direction for future research is to incorporate delay in the reception of feedback signals and in the adaptation of the source traffic rate.

Open Access This article is distributed under the terms of the Creative Commons Attribution Noncommercial License which permits any noncommercial use, distribution, and reproduction in any medium, provided the original author(s) and source are credited.

\section{References}

Adan I, van Doorn E, Resing J, Scheinhardt W (1998) Analysis of a single server queue interacting with a fluid reservoir. Queueing Syst 29:313-336 
Ahn S, Ramaswami V (2005) Steady state analysis of finite fluid flow models using finite QBDs. Queueing Syst 49:223-259

Anick D, Mitra D, Sondhi M (1982) Stochastic theory of a data-handling system with multiple sources. Bell Syst Tech J 61:1871-1894

daSilva Soares A, Latouche G (2005) A matrix-analytic approach to fluid queues with feedback control. Int J Simul Syst Sci Technol 6:4-12

daSilva Soares A, Latouche G (2006) Matrix-analytic methods for fluid queues with finite buffers. Perform Eval 63:295-314

van Doorn E, Jagers A, de Wit J (1988) A fluid reservoir regulated by a birth death-process. Stoch Models 4:457-472

Floyd S (1994) TCP and explicit congestion notification. ACM Comput Commun Rev 24:10-23

Floyd S, Jacobson V (1993) Congestion gateways for packet networks. IEEE/ACM Trans Netw 1:397-413

Gribaudo M, Telek M (2007) Stationary analysis of fluid level dependent bounded fluid models. Perform Eval 65:241-261

IEEE Standard 802.3. (1998) Carrier sense multiple access with collision detection (CSMA/CD) access method and physical layer specification, Annex 31B

Kosten L (1984) Stochastic theory of a data handling systems with groups of multiple sources. In: Rudin H, Bux W (eds) Performance of computer communication systems. Elsevier, Amsterdam, pp 321-331

Malhotra R, van Haalen R, de Man R, van Everdingen M (2002) Managing SLAs for metropolitan ethernet networks. Bell Labs Tech J 8:83-95

Malhotra R, van Haalen R, Mandjes M, Núñez-Queija R (2005) Modeling the interaction of IEEE 802.3x hop-by-hop flow control and TCP end-to-end flow control. In: Proceedings of the next generation internet networks, pp 260-267

Malhotra R, Mandjes M, Scheinhardt W, van den Berg JL (2008) Design issues of a back-pressure-based congestion control mechanism. (in preparation)

Mandjes M, Mitra D, Scheinhardt W (2003) Models of network access sing feedback fluid queues. Queueing Syst 44:365-398

Noureddine W, Tobagi F (1999) Selective back-pressure in switched Ethernet LANs. Glob Telecommun Conf 2:1256-1263

Ramanan K, Weiss A (1997) Sharing bandwidth in ATM. In: Proceedings of allerton conference, pp 732-740

Rogers L (1994) Fluid models in queueing theory and Wiener-Hopf factorization of Markov chains. Ann Appl Probab 4:390-413

Scheinhardt W, van Foreest N, Mandjes M (2005) Continuous feedback fluid queues. Oper Res Lett 33:551559 